\title{
ДВУХУРОВНЕВЫЙ МЕТОД ДИАГНОСТИКИ ТЕХНИЧЕСКОГО СОСТОЯНИЯ ШТАНГОВЫХ НАСОСНЫХ УСТАНОВОК
}

\author{
Бахтизин Рамиль Назифович1, \\ Ramil_Bahtizin@mail.ru \\ Уразаков Камил Рахматуллович1, \\ Urazakk@mail.ru \\ Исмагилов Салават Фаритович 1 , \\ salavatf@mail.ru \\ Давлетшин Филюс Фанизович2, \\ felix8047@mail.ru \\ 1 Уфимский государственный несртяной технический университет, \\ Россия, 450062, г. Уфа, ул. Космонавтов, 1. \\ 2 Башкирский государственный университет, \\ Россия, 450076, г. Уфра, ул. 3. Валиди, 32.
}

Актуальность. Значительное число крупнейших нефртяных месторождений в России находится на завершающей стадии разработки, характеризующейся снижением объемов добьчи, увеличением доли осложненного фонда скважин. Одним из наиболее распространенных способов эксплуатации скважин малодебитного фоннда являются штанговые насосные установки. Эксплуатация штанговых установок в осложненных условиях эксплуатации в ряде случаев сопровождается снижением межремонтного периода работы, повышением удельных энергетических и экономических затрат при добыче. В этих условиях одной из наиболее актуальных является задача обеспечения рентабельной разработки скважин за счет своевременной диагностики технического состояния и условий работы насосного оборудования.

Объекты: штанговая насосная установка, работающая в нефтяных добывающих скважинах, в том числе при наличии осложняющих факторов, и устьевая динамограмма работы штанговой установки, отражающая техническое состояние и условия работы внутрискважинного оборудования.

Цель: разработка нового подхода к диагностике состояния штанговых насосных установок по динамограмме, базирующаяся на решении обратных задач динамики штанговой установки.

Методы: методы численного решения уравнений в частных производных для прямых задач, включающих моделирование теоретической динамограммы работы штанговой установки; методы решения обратных задач, направленных на определение параметров модели, характеризующих работу штанговой установки.

Результаты. Разработан двухуровневый метод диагностирования состояния штанговых насосных установок по динамограмме. Предлагаемый метод диагностики включает: на первом уровне обработку практических динамограмм системой распознавания образов и на втором уровне - количественное определение последствий неисправностей решением задачи динамики штанговой установки, исходя из физических законов формирования конфигурации динамограмм. Путем анализа конфигурации динамограмм при эксплуатации насосного оборудования (нормальная работа, высокое содержание газа на приеме насоса, утечки в нагнетательном клапане насоса, низкая посадка плунжера в цилиндре) показаны примеры решения задач количественной диагностики и выдачи рекомендаций по корректировке технологического режима на основе разработанного алгоритма.

\section{Ключевые слова:}

Штанговая насосная установка, динамограмма, моделирование динамограмм,

диагностика, давление на приеме, осложняющие факторы, количественная оценка.

\section{Введение}

Значительная часть добывающих скважин в мире, относящаяся к механизированному фонду, оборудована штанговыми насосными установками (УСШН). Добыча нефти штанговыми насосными установками играет важную роль при эксплуатации малодебитных скважин, число которых по мере истощения запасов крупнейших месторождений растет.

В процессе эксплуатации скважин влияние осложняющих факторов может привести к снижению дебита скважины, межремонтного периода работы, повышению экономических затрат при добыче. Оперативная диагностика осложнений и неисправностей поз- воляет своевременно избежать ненормального развития событий, выявить потенциальные скважины, работающие в нерентабельной области технологического режима с целью последующего проведения мероприятий по оптимизации режима работы данной группы скважин.

Одним из наиболее распространенных методов диагностики технического состояния и условий работы насосного оборудования является анализ и интерпретация конфигурации устьевой динамограммы. Разработан ряд методов и алгоритмов автоматизированной диагностики УСШН по динамограмме, базирующихся на системах распознавания образов. 
На основе данной технологии диагностика внутрискважинного оборудования может рассматриваться как процесс распознавания образов с извлечением признаков, определенных по форме фактической динамограммы, и диагностикой на основе определенной модели. Среди наиболее распространенных методов и подходов, используемых при распознавании образов фактических динамограмм, следует выделить: вейвлетный анализ, анализ частотного спектра динамограмм, анализ отклонения фактической динамограммы от эталонной, выделение релевантных точек динамограммы, анализ признаков динамограмм на базе рядов Фурье [1-6]. Для распознавания образов фактических динамограмм, прогнозирования технического состояния штанговых установок широкое распространение получил метод нейронных сетей. Метод использует базы данных, включающие определенное количество известных классов динамограмм, причем динамограммы с аналогичной конфигурацией сгруппированы в один класс. После настройки на обучающей выборке динамограмм нейронная сеть позволяет определять принадлежность к классам новых, диагностируемых данных. При использовании в качестве обучающей выборки последовательности динамограмм, отражающих динамику изменения нагрузок на полированный шток во времени в процессе эксплуатации определенной скважины, известные методики на базе нейронных сетей позволяют также спрогнозировать конфигурацию последующих во времени динамограмм [7-11].

В настоящее время при разработке систем диагностирования штанговых установок значительный акцент делается на нейросетевом подходе. Следует, однако, отметить, что рассмотренные выше диагностические системы, основанные на методах распознавания образов, в частности нейронные сети, направлены преимущественно на качественную диагностику штанговых установок. С точки зрения повышения информативности результатов диагностики интерес представляет разработка алгоритмов диагностики, базирующихся на математическом моделировании работы штанговых установок. Разработке принципов построения алгоритмов диагностики и прогнозирования технического состояния штанговых установок, основанных на численном и аналитическом решении волнового уравнения, моделировании, посвящены фундаментальные работы американских и советских исследователей: С.Г. Гиббса А.Б. Неели, А.С. Вирновского [12, 13]. В последнее время усиливается интерес к данному подходу, что отражено в ряде опубликованных работ. Использование фактических динамограмм для расчета конкретных количественных параметров штанговых установок, в частности, эффективного хода плунжера путем пересчета устьевой динамограммы в плунжерную, рассмотрено в работе [14]. Прогнозирование работы плунжера штангового насоса базируется на методе рядов Фурье (FSM - Fourier series method) решения волнового уравнения колебаний штанговой колонны. По величинам перемещения текущего сечения штанговой колонны (смещения) и нагрузки в этом сечении, например, полированного штока, с помощью FSM получают смещение и нагрузку в нижней части штанго- вой колонны, соответствующей плунжеру насоса. FSM применяется для решения различных задач проектирования и эксплуатации штанговых установок. Моделированию физических процессов при штанговонасосной эксплуатации с учетом конкретных условий рассматриваемого месторождения посвящены работы $[15,16]$. Разработка комплексной системы проектирования и автоматического контроля работы системы пластскважина-насосная установка, позволяющей оптимизировать процесс эксплуатации и обеспечить максимальную рентабельность добычи нефти, рассмотрена в [17].

Таким образом, математическое моделирование работы штанговых установок в осложненных условиях эксплуатации с детальным учетом физического влияния осложняющих факторов на формирование динамических нагрузок в рассматриваемой системе позволяет осуществлять детальную диагностику технического состояния штанговых установок, включающую не только качественное выявление наличия неисправностей или влияния осложняющих факторов в работе оборудования, но и определение степени их проявления, количественных параметров, характеризующих каждый из осложняющих факторов эксплуатации, а также выработать конкретные количественные рекомендации по оптимизации режима с учетом индивидуальных параметров работы адресной скважины.

Для решения данной задачи и повышения информативности результатов диагностики предлагается двухуровневый метод диагностирования штанговых насосных установок по фактической динамограмме, включающий обработку практических динамограмм системой распознавания образов на первом уровне, и количественную оценку неисправностей исходя из физических законов формирования конфигурации динамограмм решением задачи динамики штанговой установки на втором уровне (рис. 1).

Для реализации предложенного метода разработан алгоритм, включающий в себя следующий комплекс операций:

1. Строится теоретическая динамограмма нормальной работы насоса по геолого-техническим и технологическим параметрам скважины.

2. Путем сопоставления фактической и теоретической динамограмм нормальной работы насоса по экспертной системе, основанной на методе распознавания образов, определяется вероятный тип неисправности.

3. В соответствии с выбранным типом (выбранными типами) неисправности, путем последовательного варьирования коэффициентов (параметров), характеризующих осложнения и неисправности в математической модели, строится теоретическая динамограмма с учетом осложнений/неисправностей, наиболее близкая к фактической, при этом количественно оцениваются причины и факторы, вызывающие осложнения и неисправности.

Построение теоретической динамограммы выполняется с учетом данных инклинометрии, замеров устьевого давления и затрубного давления газа, динамического уровня жидкости. 


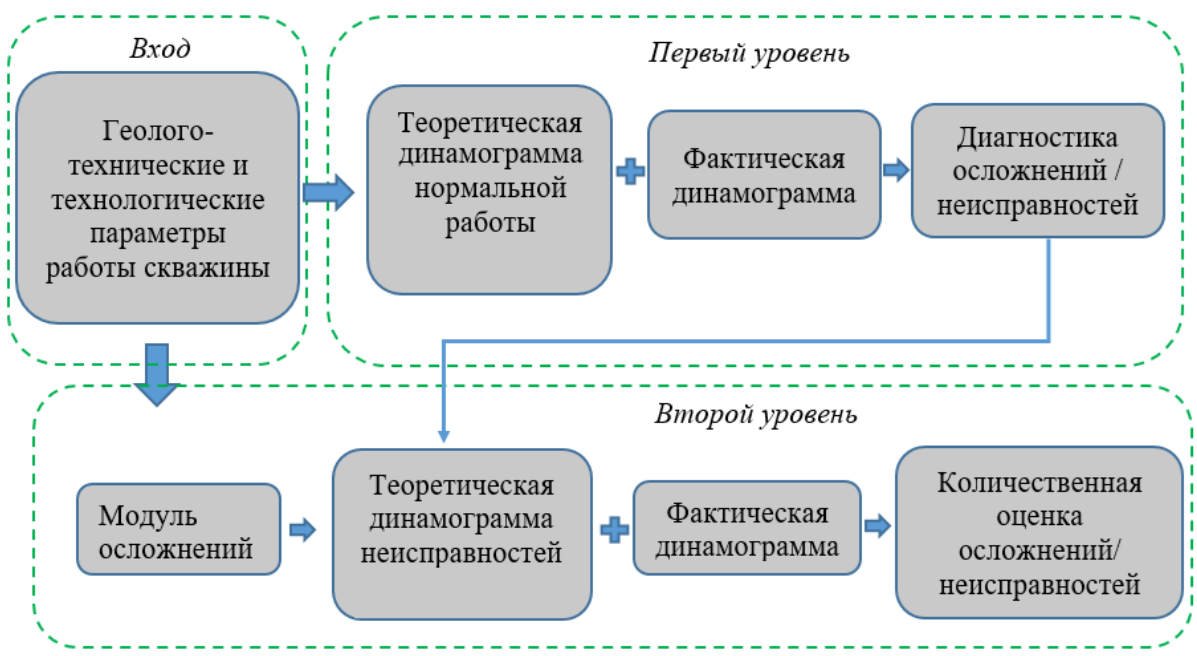

Рис. 1. Общая схема алгоритма диагностики

Fig. 1. General scheme of the diagnostic algorithm

\section{Математическая модель}

Моделирование динамических нагрузок, действующих на штанговую колонну и плунжер насоса, основывается на уравнении продольной упругой волны с учетом нагрузок, обусловленных граничным трением колонны штанг о стенки НКТ, вязким трением о жидкость, а также переменного давлением в полости под плунжером [18-20]:

$$
\left\{\begin{array}{l}
\rho_{s} S_{r} \frac{\partial^{2} u}{\partial t^{2}}=E S_{r}\left(\frac{\partial^{2} u}{\partial x^{2}}\right)+\left(\rho_{s}-\rho_{t}\right) S_{r} g \cos \alpha- \\
\quad-\frac{P \mu_{f g}(\mu) \rho_{t}}{2}\left(\frac{\partial u}{\partial t}+w_{t}\right) \mid \frac{\partial u}{\partial t}+w_{t}\left(\left(1+\frac{l_{e k v}}{l_{r}}\right)-f N \operatorname{sign}(v) ;\right. \\
u(0, t)=\frac{S}{2} \cos (2 \pi n t) ; \\
E S_{r} \frac{\partial u}{\partial x}(L, t)=\left(p_{\text {out }}-p_{p}\right) S_{p}-\mu_{p}\left(1,65 \frac{D_{p}}{\delta}-127\right) \frac{\partial u}{\partial t}(L, t),
\end{array}\right.
$$

где $u(x, t)$ - перемещение фиксированной точки колонны штанг; $t$ - время; $E$ - модуль Юнга материала штанг; $\rho_{s}$ - плотность материала штанг; $x$ - лагранжева координата по длине колонны штанг; $\rho_{t}-$ плотность смеси в НКТ; $g$ - ускорение свободного падения; $\alpha$ - угол наклона скважины к вертикали в данной точке НКТ; $P$ - периметр штанги; $S_{r}$ - площадь поперечного сечения штанг; $\mu_{f g}$ - переменный коэффициент сопротивления жидкости движению штанг; $\mu$ динамическая вязкость; $w_{t}(x, t)-$ скорость движения газожидкостной смеси в НКТ; $f$ - коэффициент трения штанг о НКТ; $N$ - удельная прижимающая сила; $\operatorname{sign}(v)$ - функция, определяющая направление силы граничного трения; $v$ - скорость движения штанг в данной точке штанговой колонны; $S$ - длина хода полированного штока; $n$ - число качаний балансира; $L$ - длина штанговой колонны; $p_{\text {out }}$ - давление на выкиде насоса; $S_{p}$ - площадь поперечного сечения плунжера; $\mu_{p}-$ коэффициент трения в плунжерной паре; $D_{p}$ - диаметр плунжера; $\delta$ - зазор между плунжером и цилиндром насоса; $p_{p}$ - давление в подплунжерной полости насоса; $\rho_{p}-$ плотность смеси в под- плунжерной полости насоса; $l_{r}$ - длина насосной штанги; $l_{e k v}$ - эквивалентная по трению длина насосных штанг. Эквивалентная длина штанги - длина фиктивной штанги, заменяющей по действию сил гидродинамического трения одну штанговую муфту. $\mathrm{C}$ помощью данного понятия учитывается сопротивление, оказываемое со стороны жидкости движению штанговых муфт.

В предложенной математической модели осложняющие факторы являются слагаемыми исходной системы уравнений. В частности, силы граничного трения, развиваемые в искривленных и наклонных участках ствола скважины, и вязкого трения, действующие при подъеме продукции скважины с высокой вязкостью, являются распределенными силами в уравнении, описывающем динамику штанговой колонны. Утечки в клапанах насоса приводят к возникновению потоков через клапаны и влияют на скорость изменения давления в подплунжерной полости насоса. Локальное повышение коэффициента граничного трения при заедании или заклинивании плунжера в цилиндре приводит к значительному росту силы граничного трения в плунжерной паре. Относительное положение клетки всасывающего клапана, посадочного корпуса вставного насоса и плунжера определяет возможность возникновения ударов плунжера в цилиндре. Таким образом, осложняющие факторы в математической модели характеризуются комплексом физико-химических (доля газа на приеме насоса, коэффициент граничного трения, вязкость откачиваемой продукции и др.), стереометрических и геометрических (кривизна ствола скважины, положение плунжера относительно цилиндра насоса в процессе его хода, эффективная площадь проходного сечения клапанов насоса или лифтовых труб и др.) параметров $[21,22]$.

С использованием разработанной математической модели путем численного решения системы (1) проведен расчет теоретических динамограмм для выборки реальных скважин. Апробация модели показала хорошее согласие между фактическими данными и результатами моделирования (рис. 2). 

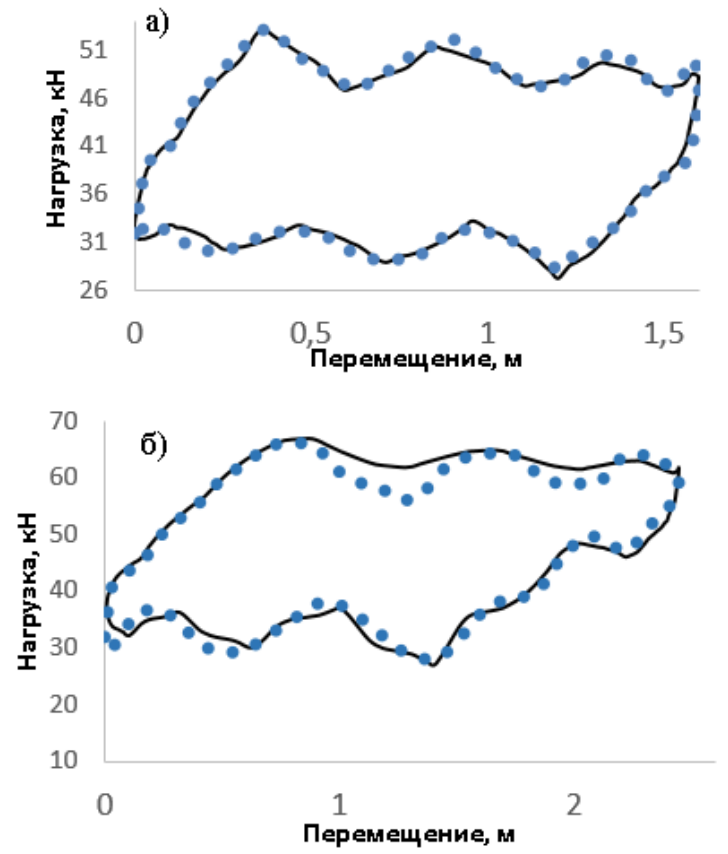

Pис. 2. Фактические и расчетные динамограммы: а) скв. № 88 Культюбинского месторождения; б) скв. № 116 Шакшинского месторождения (точки измеренные значения, сплочная линия - расчетная динамограмма)

Fig. 2. Actual and calculated dynamograms: a) well no. 88, Kultyubinskoe field; б) well no. 116, Shakshinskoe field (points - measured values, solid line calculated dynamogram)

\section{Экспертная система диагностики состояния штанговых установок}

Экспертная система предназначена для программно-алгоритмизированного диагностирования типа неисправности в работе штанговой установки по известным параметрам реальной скважины и фактической динамограмме. На первом этапе определены 13 классов осложнений и неисправностей, включающих нормальную работу насоса и работу насоса с влиянием осложняющих факторов, обуславливающих изменение конфигурации динамограмм. Распознавание образов фактических динамограмм базируется на применении диагностирующих признаков, характеризующих конфигурацию фактических динамограмм на различных участках. Для теоретической динамограммы используется обозначение АБВГ, где линия АБ соответствует линии восприятия штангами веса жидкости в насоснокомпрессорных трубах, линия ВГ - линия разгрузки насосных штанг. Диагностические признаки:

1. Фактическая динамограмма расположена ниже линии БВ теоретической динамограммы

2. Существование площади над линией АГ теоретической динамограммы, которая больше площади под линией АГ.

3. «Всплеск» нагрузок в правой верхней части динамограммы.

4. Смещение точек фактической динамограммы в конце линии разгрузки насосных штанг.

5. Смещение точек фактической динамограммы в конце линии восприятия нагрузки насосными штангами.

6. Минимальная нагрузка по фактической динамограмме значительно меньше нагрузок по теоретической динамограмме.

7. Смещение точек фактической динамограммы относительно линии разгрузки штанг теоретической динамограммы.

8. Наличие петли в конце хода вверх.

9. Наличие петли в конце хода вниз.

10. Характерная конфигурация линии разгрузки штанг «пистолет».

11. «Всплеск» нагрузок в левой нижней части динамограммы.

12. «Закругление» формы динамограммы.

13. Длина линии нагрузки насосных штанг значительно превышает длину линии разгрузки.

14. Длина линии разгрузки насосных штанг значительно превышает длину линии восприятия нагрузки.

Наличие вышеперечисленных признаков у эталонных динамограмм, каждая из которых характеризует свой вид неисправности, приведена в таблице (1 признак имеет место, 0 - признак отсутствует, «-» признак не характерен).

Таблица. Классификация динамограмм по характерным признакам

Table.

Characterization of dynamograms

\begin{tabular}{|l|c|c|c|c|c|c|c|c|c|c|c|c|c|c|}
\hline \multicolumn{1}{|c|}{ Признак/тип неисправности/Features/fault type } & 1 & 2 & 3 & 4 & 5 & 6 & 7 & 8 & 9 & 10 & 11 & 12 & 13 & 14 \\
\hline Нормальная работа насоса/Normal operation of the pump & 0 & 0 & 0 & 0 & 0 & 0 & 0 & 0 & 0 & 0 & 0 & 0 & 0 & 0 \\
\hline $\begin{array}{l}\text { Срыв подачи от утечки в нагнетательном клапане } \\
\text { Leakage in the traveling-valve, loss of feed pump }\end{array}$ & 1 & 1 & 0 & 0 & 1 & - & 0 & 0 & 0 & 0 & 0 & 0 & 0 & 0 \\
\hline Обрыв штанг или отворот плунжера/Rod break or plunger lapel & 1 & 0 & - & 0 & 1 & - & - & 0 & 0 & 0 & 0 & 0 & 0 & 0 \\
\hline Высокая посадка плунжера/Pump hitting up, spaced too high & 0 & - & 1 & 1 & - & 0 & 1 & 0 & 0 & 1 & 0 & 0 & 0 & 0 \\
\hline Заедание плунжера в конце хода вверх/Jamming of the plunger at the top & 0 & - & 1 & - & 0 & 0 & 1 & 0 & 0 & 0 & 0 & 0 & 0 & 0 \\
\hline Заклинивание плунжера/Jamming of the plunger & 0 & 0 & - & 1 & 1 & 1 & - & 0 & 0 & 1 & 0 & 0 & 0 & 0 \\
\hline Прихват плунжера/Sticking of the plunger & 0 & - & - & 1 & 1 & - & - & 0 & 1 & - & 0 & 0 & 0 & 0 \\
\hline Низкая посадка плунжера/Pump hitting down/spaced too low & 0 & - & 0 & 0 & - & - & - & 0 & 1 & 0 & 1 & 0 & 0 & 0 \\
\hline Влияние газа на приеме насоса/Heavy gas interference & 0 & - & 0 & 1 & 0 & - & - & 0 & 0 & 1 & 0 & 0 & 0 & 1 \\
\hline Заедание плунжера в конце хода вниз/Jamming of the plunger at the down & 0 & - & 0 & 0 & 0 & 0 & 0 & 0 & 0 & 0 & 1 & 0 & 0 & 0 \\
\hline $\begin{array}{l}\text { Высокая вязкость откачиваммй продукции } \\
\text { Нigh visсовitу оf thе рuтреd рroduct }\end{array}$ & 0 & 0 & 0 & 0 & 0 & 1 & 0 & 0 & 0 & 0 & 0 & 1 & 0 & 0 \\
\hline Утечки в нагнетательной части насоса/Traveling-valve leak & 0 & 0 & 0 & 0 & 1 & 0 & 0 & 0 & 0 & 0 & 0 & 0 & 1 & 0 \\
\hline Утечки в приемной части насоса/Standing-valve leak & 0 & 0 & 0 & 1 & 0 & 0 & 0 & 0 & 0 & 0 & 0 & 0 & 0 & 1 \\
\hline
\end{tabular}




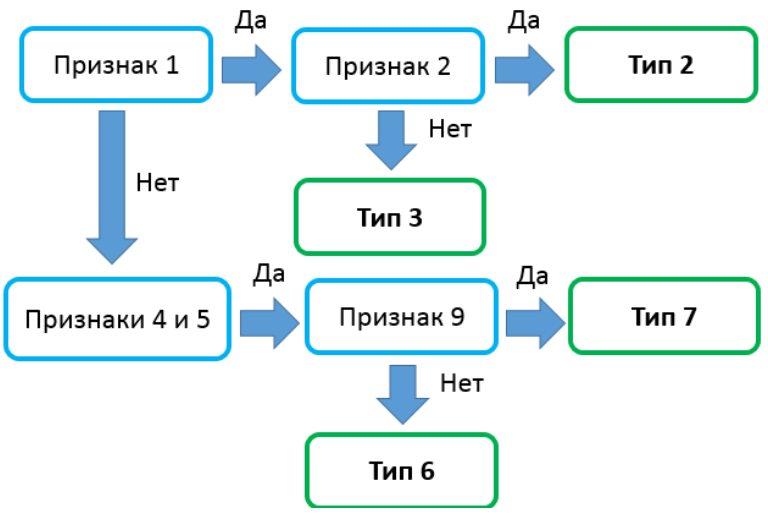

Рис. 3. Блок-схема 1 алгоритма распознавания

Fig. 3. Block-diagram 1 of recognition algorithm

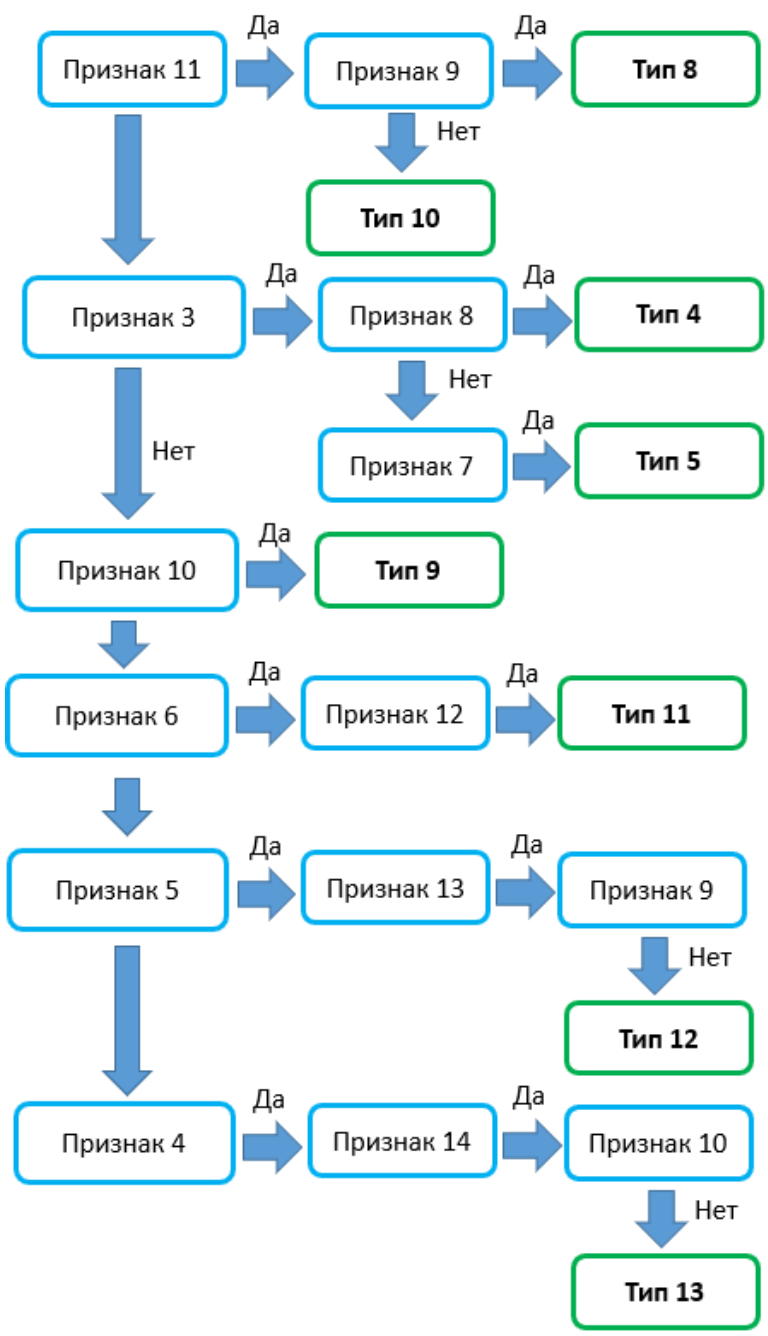

Рис. 3. Блок-схема 2 алгоритма распознавания

Fig. 3. Block-diagram 2 of recognition algorithm

Блок-схем алгоритма распознавания представлена на рис. 3, 4. На первом этапе проводится оценка признаков в рамках первой части блок-схемы (рис. 3) для диагностики осложнений и неисправностей в работе штанговой установки, определяющих формирование конфигурации динамограммы практически на всех ее участках: обрыв штанг/отворот плунжера, срыв подачи от утечек в нагнетательной части, заклинивание и заедание плунжера. Далее выполняется оценка признаков для диагностики осложнений и неисправностей, определяющих конфигурацию определенного участка динамограммы (рис. 4). Анализ различных участков динамограммы путем последовательной оценки признаков 3-6, 11 позволяет выявить несколько осложнений и неисправностей, вносящих вклад в конфигурацию динамограммы на определенном ее участке.

\section{Диагностика степени проявления осложняющих факторов по динамограмме}

На втором уровне диагностической системы осуществляется количественная оценка осложнений и неисправностей исходя из физических законов формирования конфигурации динамограмм путём решения задачи динамики штанговой установки. Приближение теоретической динамограммы к фактической достигается варьированием числовых значений коэффициентов (параметров), характеризующих типы неисправностей, выявленных на первом уровне экспертной системой.

Принципы количественной диагностики состояния штанговой установки рассматриваются на примере осложнений «влияние газа на приеме» и «утечки в клапанах». Оба класса осложнений оказывают влияние на динамику изменения давления в подплунжерной полости насоса, а значит, определяют и изменение дифференциальной нагрузки на плунжер насоса в процессе его хода, что отражается в конфигурации устьевой динамограммы.

Влияние газа на динамограмму в значительной степени зависит от особенностей протекания процессов в подплунжерной полости насоса при откачке многофазной смеси. При расчете динамики давления в подплунжерной полости с учетом влияния газа существует два предельных случая: при значительной скорости всплытия газовых пузырьков в нефти, когда газовая фаза успевает отделиться от нефтяной, растворением газа в нефти можно пренебречь, и изменение давления будет характеризоваться сжатием и расширением газа в полости насоса. При этом динамика давления в подплунжерной полости будет определяться, прежде всего, долей газа на приеме насоса и объемом вредного пространства насоса:

$$
\begin{gathered}
\frac{d p_{p}}{d t}=-\frac{\rho_{g}}{V_{g}}\left(\frac{d \rho_{g}}{d p}\right)^{-1} \times \\
\times\left(\begin{array}{l}
\frac{V_{l}}{V_{p}} \frac{d V_{p}}{d t}-\sqrt{2}\left(1-\alpha_{\text {in }}\right) \lambda_{\text {in }} \delta_{\text {in }} \frac{p_{\text {in }}-p_{p}}{\sqrt{\rho_{l}\left|p_{\text {in }}-p_{p}\right|}}- \\
-\sqrt{2}\left(1-\alpha_{\text {out }}\right) \lambda_{\text {out }} \delta_{\text {out }} \frac{p_{\text {out }}-p_{p}}{\sqrt{\rho_{l}\left|p_{\text {out }}-p_{p}\right|}}
\end{array}\right),(2)
\end{gathered}
$$

где $\rho_{g}, V_{g}$ - плотность и объем свободного газа в подплунжерной полости насоса в предположении, что газ не растворяется/выделяется из жидкости; $\alpha_{\text {in }}$ - доля газа на приеме насоса; $\rho_{l}, V_{l}$ - плотность и объем жидкости соответственно; $p_{\text {in }}$ - давление на приеме 
насоса; $V_{p}$ - объем подплунжерной полости насоса; $\lambda$ - коэффициент расхода, индекс in соответствует всасывающему клапану, индекс out - нагнетательному; $\delta$ - площадь проходного сечения клапана, индекс in соответствует всасывающему клапану, индекс out нагнетательному.

При небольшой скорости всплытия пузырьков газа, при которой пузырьки газа равномерно распределяются в нефти, будут наблюдаться равновесные процессы выделения и растворения газа в нефти и изменение во времени давления в полости насоса будет также определяться растворимостью газа в нефти

$$
\frac{d p_{p}}{d t}=\frac{1}{V_{p}}\left(\begin{array}{l}
\sqrt{2 \rho_{\text {in }}} \lambda_{\text {in }} \frac{p_{\text {in }}-p_{p}}{\sqrt{p_{\text {in }}-p_{p}}} k_{\text {in }} \delta_{\text {in }}+ \\
+\sqrt{2 \rho_{\text {out }}} \lambda_{\text {out }} \frac{p_{p}-p_{\text {out }}}{\sqrt{p_{p}-p_{\text {out }}}} k_{\text {out }} \delta_{\text {out }}+ \\
+\rho_{p}(t) \frac{d V_{p}}{d t}
\end{array}\right)\left(\frac{d \rho_{p}}{d p}\right)^{-1},
$$

где $\rho_{p}-$ плотность смеси в подплунжерной полости насоса; $k \in[0 ; 1]$ - коэффициент, определяющий степень открытия клапанов насоса.

Фактическая и расчетные динамограммы работы штангового насоса, полученные путем численного решения системы уравнений (1)-(3), представлены на рис. 5.

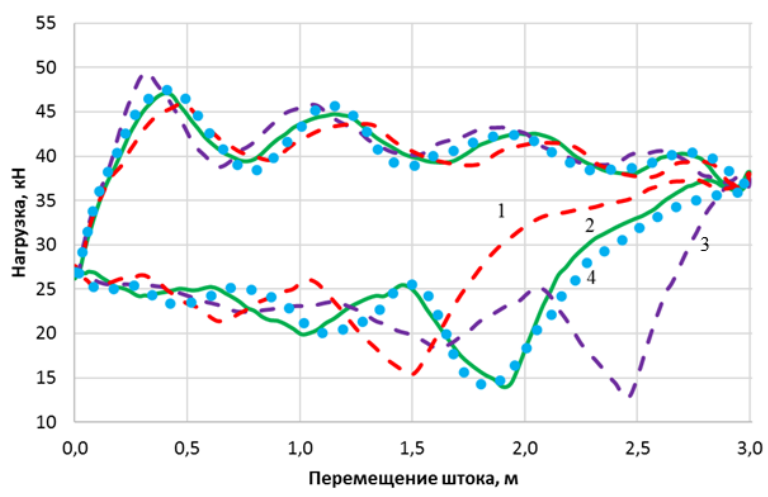

Pис. 5. Динамограммы работы итангового насоса с учетом влияния газа (штриховая линия 1 - модельная динамограмма при $\alpha_{i n}=0,45$, сплошная линия 2 -модельная динамограмма при $\alpha_{\text {in }}=0,25$, итриховая линия 3 - модельная динамограмма при $\alpha_{i n}=0,05$, точки 4 - фактическая динамограмма)

Fig. 5. Dynamometer cards of the pump operation, taking into account the influence of gas (dashed line 1 model dynamometer card with $\alpha_{i n}=0,45$, solid line 2 - model dynamometer card with $\alpha_{i n}=0,25$, dashed line 3 - model dynamometer card with $\alpha_{i n}=0,05$, points 4 - actual dynamometer card)

Из графиков на рис. 5 следует, что за счет сжимаемости газа по мере возрастания газосодержания в продукции скважины увеличивается длина линии снятия нагрузки и повышается ее наклон. При малой величине объемного содержания газа на приеме длина линии снятия нагрузки минимальна и практически соответствует линии разгрузки, обусловленной статической деформацией штанговой колонны (кривая 3 , рис. 5), при значительном газо-содержании (кривая 1, рис. 5) скорость роста давления в подплунжерной полости существенно снижается, и нагрузка на головку балансира также снижается значительно более медленно.

При наличии нескольких осложняющих факторов, каждый из которых оказывает влияние на формирование динамических нагрузок в штанговой колонне, результирующая конфигурация динамограммы определяется совместным действием факторов. Рассмотрим одновременное наложение осложнений «утечки в нагнетательном клапане» и «влияние газа» на конфигурацию устьевой динамограммы (рис. 6).

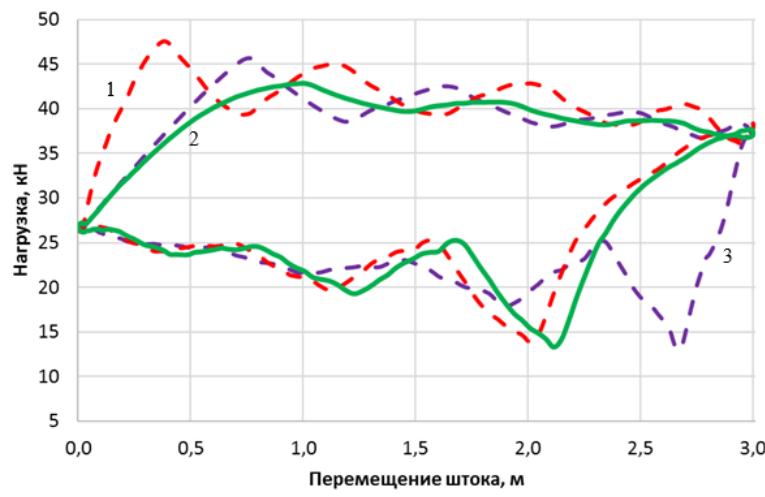

Pис. 6. Конфигурачия динамограммы при одновременном наложении осложнений «утечки в нагнетательном клапане» и «влияние газа» $(1-$ влияние газа, 2 - совместное влияние осложнений, 3 утечки в нагнетательном клапане)

Fig. 6. Configuration of the dynamometer card with simultaneous imposition of complications «leakage in the discharge valve» and "gas effect» (1 -gas influence, 2 - the combined effect of complications, 3 -leakage in the discharge valve)

Утечки в нагнетательном клапане (кривая 3, рис. 6) оказывают влияние главным образом на конфигурацию линии восприятия нагрузки на динамограмме и характеризуются увеличенным наклоном динамограммы в начале хода плунжера вверх, обусловленным оттоком откачиваемой смеси из колонны лифтовых труб в подплунжерную полость, приводящим к снижению темпов изменения давления в подплунжерной полости. Напротив, влияние газа (кривая 1, рис. 6) приводит к изменению конфигурации линии снятия нагрузки и отражается в увеличении наклона кривой при ходе вниз за счет высокой сжимаемости газа. При одновременном действии этих осложнений устьевая динамограмма характеризуется увеличенным наклоном как линии снятия, так и линии восприятия нагрузки (кривая 2, рис. 6). Поскольку каждое из рассматриваемых осложнений вносит вклад в конфигурацию динамограммы на определенном участке, количественная диагностика по динамограмме позволяет одновременно рассчитать параметры, характеризующие оба осложнения, в частности, долю газа на приеме насоса, потери подачи насоса за счет сжатия 
газа в подплунжерной полости, объемный расход смеси через нагнетательный клапан в ходе утечки (дебит утечек). Полученная информация является основой для принятия решений и рекомендаций по последующей оптимизации режима работы штанговой установки, включающей: изменение скорости откачки при превышении максимального допустимого объемного содержания газа на приеме насоса и существенной потере подачи за счет влияния газа; промывку скважины или подъем насоса при чрезмерной величине потери подачи насоса за счет утечек в нагнетательной части насоса и др.

Значительный интерес представляет расчет эффективной вязкости смеси в стволе скважины при высокой вязкости откачиваемой продукции. Под эффективной вязкостью понимается осредненная по стволу скважины величина вязкости, значение которой можно использовать для адекватного учета сил вязкого трения при проектировании технологического режима штанговых установок. На рис. 7 приведен расчет нагрузок на полированный шток при различной величине эффективной вязкости откачиваемой продукции. Из рисунка видно, что при прочих равных условиях с увеличением вязкости происходит увеличение максимальной и снижение минимальной нагрузки на полированный шток, при этом наблюдается «закругление» формы динамограммы, характеризуемое плавным изменением нагрузок в процессе хода полированного штока.

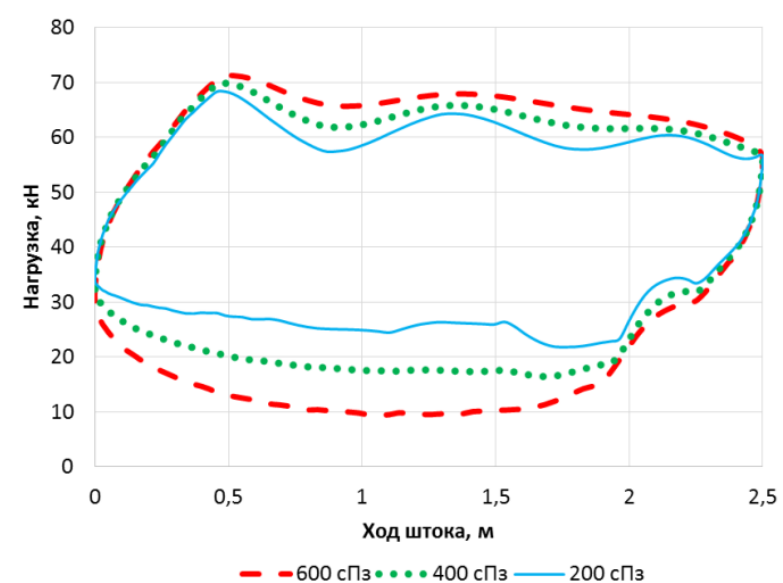

Pис. 7. Динамические нагрузки в точке подвеса итанговой колонны при высокой вязкости откачиваемой продукиии (сплошная линия соответствует эффективной вязкости $\mu_{o}=200$ сПз, точечная $\mu_{o}=400 \mathrm{c} \Pi_{3}$, итриховая линия $\left.-\mu_{o}=600 \mathrm{c} \Pi_{3}\right)$

Fig. 7. Dynamic loads at the point of suspension of the rod column at high viscosity of the pumped product (solid line corresponds to effective viscosity $200 \mathrm{sPz}$, scatter-400 sPz, dashed line - $600 \mathrm{sPz}$ )

\section{Оптимизация технологического режима штанговой установки}

Разработка конкретных количественных рекомендаций по оптимизации режима работы штанговой установки рассматривается на примере осложнений в работе штангового насоса, связанных с возникновением ударов плунжера в цилиндре о клетку всасыва- ющего клапана в конце хода плунжера вниз при низкой посадке плунжера.

Дополнительное растяжение штанг в конце хода вниз, обусловленное динамическими составляющими нагрузок на штанговую колонну, может привести к возникновению ударов плунжера о клетку всасывающего клапана. При посадке плунжера на клетку клапана происходит передача части веса штанговой колонны насосно-компрессорным трубам, в результате нагрузка на головку балансира снижается (рис. 8)

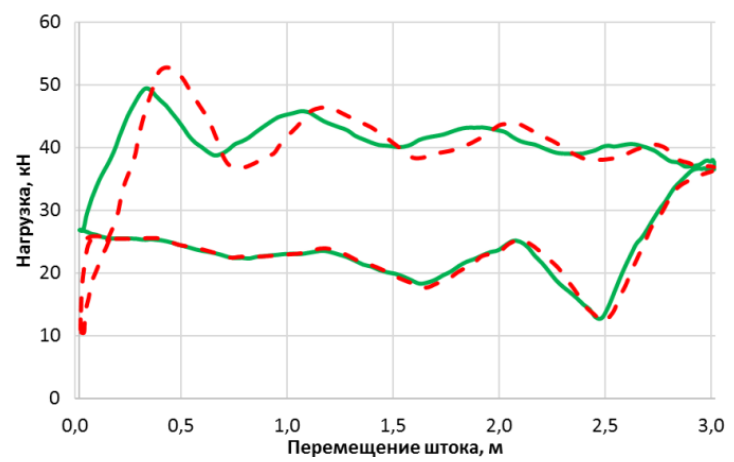

Pис. 8. Моделирование работы насоса при низкой посадке (итриховая линия - динамограмма при наличии удара плунжера о клетку всасывающего клапана в конце хода вниз, сплошная - после корректировки подвески плунжера)

Fig. 8. Simulation of pump operation at a low fit (dashed line - dynamogram with pump hitting down at spaced too low, solid line - after adjusting the plunger suspension)

Моделирование удара плунжера осуществляется с учетом геометрии цилиндра насоса, а именно, месторасположения клетки всасывающего клапана. Удар возникает при условии, что перемещение плунжера в цилиндре превышает величину, соответствующую глубине расположения клетки клапана. Разгрузка штанговой колонны балансира моделируется в соответствии с законом Гука - в конце хода вниз перемещение головки балансира опережает перемещение плунжера, за счет чего штанги сокращаются, сила упругого растяжения штанг снижается, обуславливая снижение нагрузок на головку балансира. В рамках разработанного алгоритма количественная диагностика для данной задачи сводится к расчету теоретических динамограмм, моделирующих удар плунжера о клетку всасывающего клапана, и их приближения к фактической динамограмме, посредством чего определяется параметр, соответствующий величине расстояния, проходимой полированным штоком после удара плунжера. Корректировка подвески плунжера на величину найденной поправки позволяет исключить в последующем удары плунжера в цилиндре насоса. На модельном примере (рис. 8) показано, как корректировкой глубины подвески плунжера в цилиндре насоса возможно устранение удара плунжера о клетку всасывающего клапана, позволяющего значительно повысить надежность и ресурс работы штангового насоса и его клапанов, а также увеличить эффективный ход плунжера в цилиндре. 


\section{Выводы}

1. Разработан двухуровневый метод диагностирования состояния скважинных насосных установок: а) на первом уровне экспертной системой, основанной на методе распознавания образов, выявляются неисправности в работе скважинной насосной установки, отраженные на фактической динамограмме, и в соответствии с выявленными характерными признаками относятся к определенному классу неисправности. Характерные по конфигурации динамограммы ранжированы на 13 классов, включая нормальную работу насоса и работу насоса с осложнениями, вызывающими изменение формы динамограмм; б) на втором шаге осуществляется количественная оценка осложнений и неисправностей, путем итеративного расчета теоретических динамограмм, соответствующих классу, определенному на первом этапе, и их приближения к фактической динамограмме. Числен-

\section{СПИСОК ЛИТЕРАТУРЫ}

1. Robust technology and system for management of sucker rod pumping units in oil wells T.A. Aliev, A.H. Rzayev, G.A. Guluyev, T.A. Alizada, N.E. Rzayeva // Mechanical Systems and Signal Processing. - 2018. - V. 99. - P. 47-56.

2. Boyuan Zh., Xianwen G. Sucker rod pumping diagnosis using valve working position and parameter optimal continuous hidden Markov model // Journal of Process Control. - 2017. - V. 59. P. $1-12$.

3. Диагностирование глубинно- насосных скважин динамометрированием / Г.Г. Гилаев, Б.В. Ефименко, Л.С. Гончарова, В.С. Исупов. - Ижевск: ООО «РА «Парацельс»»», 2008. - 212 с.

4. Садов В.Б. Подход к определению установок скважинных штанговых установок по динамограмме // Нефтяное хозяйство. - 2016. - № 2. - С. 90-93.

5. A new approach to diagnosis of sucker rod pump systems by analyzing segments of downhole dynamometer cards / D.R. Galdir Jr., L. Schnitman, R. Reis, F. Mota // SPE (Society of Petroleum Engineers) - 173964, SPE Artificial Lift Conference. - Latin America and Caribbean held in Salvador, Bahia, Brazil, 27-28 May 2015. - P. 1-13.

6. Ковшов В.Д., Сидоров М.Е., Светлакова С.В. Динамометрирование, моделирование и диагностирование состояния глубинной штанговой насосной установки// Известия вузов. Нефть и газ. - 2011. - № 3. - С. 25-29.

7. Li K., Han Y., Wang T. A novel prediction method for down-hole working conditions of the beam pumping unit based on 8directions chain codes and online sequential extreme learning machine // Journal of Petroleum Science and Engineering. 2018. - V. 160. - P. 285-301.

8. Сибагатуллин Р.Р., Майский Р.А. Обобщенный алгоритм диагностики работы штанговых установок // Проблемы сбора, подготовки и транспорта нефти и нефтепродуктов. - 2017. № 3. - С. 69-77.

9. Кузьмин А.Н., Вялых И.А. Прогнозирование технического состояния штанговых глубинных насосов на основе нейросетевых технологий // Вестник Пермского национального исследовательского политехнического университета. Химическая технология и биотехнология. - 2016. - № 3 - С. 9-19.

10. Using the curve moment and the PSO-SVM method to diagnose downhole conditions of a sucker rod pumping unit $/ \mathrm{K}$. Li, G. Xianwen, T. Zhongda, Q. Zhixue // Petroleum Science. 2013. - V. 10. - P. 73-80. ное приближение теоретической динамограммы к фактической достигается варьированием числовых значений коэффициентов (параметров), характеризующих осложнения и неисправности в математической модели.

2. Разработан метод диагностики, позволяющий количественно оценивать следующие физические параметры, характеризующие степень проявления осложнений в работе УСШН: газосодержание на приеме насоса; эффективная динамическая вязкость смеси; доля подачи насоса, теряемая вследствие утечек; часть хода полированного штока, происходящая с ударом плунжера. Полученные результаты могут быть в одних случаях использованы для корректировки существующего технологического режима, а в других - для адекватного подбора типоразмера насосного оборудования и расчета планируемого режима.

11. Fault diagnosis for down-hole conditions of sucker rod pumping systems based on the FBH-SC method / K. Li, X.W. Gao, H.B. Zhou, Y. Han // Journal of Petroleum Science and Engineering. - 2015. - V. 12. - P. 135-147.

12. Вирновский А.С. Теория и практика глубинно-насосной добычи нефти. - М.: Недра, 1971. - 183 с.

13. Gibbs S.G., Neely A.B. Computer diagnosis of down-hole conditions in sucker rod pumping wells // Journal of Petroleum Technology. - 1966. - V. 1. - P. 93-98.

14. Chen Z., White L.W., Zhang H. Predicting sucker-rod pumping systems with Fourier series // SPE (Society of Petroleum Engineers) Production \& Operations. - 2018. - V. 33. P. 928-940.

15. Deep well lifting new solution in Tarim oilfield / R. Zhao, Y. Peng, Q. Li, J. Shi et al. // SPE (Society of Petroleum Engineers) 192494, SPE Middle East Artificial Lift Conference and Exhibition. - Manama, Bahrain, 28-29 November 2018. - P. 1-14.

16. Li-Ming L., Hua Zh. Application and effect of buoyancy on sucker rod string dynamics // Journal of Petroleum Science and Engineering. - 2016. - V. 146. - P. 264-271.

17. Model predictive automatic control of sucker rod pump system with simulation case study / B. Hansen, B. Tolbert, C. Vernon, J.D. Hedengren // Computers and Chemical Engineering. - 2019. V. 121. - P. 265-284.

18. Dynamic model of a Rod Pump Installation for inclined wells / R.N. Bakhtizin, K.R. Urazakov, S.F. Ismagilov, A.S. Topol'nikov, F.F. Davletshin // SOCAR Proceedings. - 2017. - № 4. - P. 74-82.

19. Расчет теоретической динамограммы с учетом осложнений в работе скважинного штангового насоса / К.Р. Уразаков, Р.Н. Бахтизин, С.Ф. Исмагилов, А.С. Топольников // Нефтяное хозяйство. - 2014. - № 1. - С. 90-93.

20. Brill J.P., Mukherjee H. Multiphase flow in wells. - Richardson, Texas: Society of petroleum engineers, 1999. - 384 p.

21. Уразаков К.Р., Белов А.Е., Давлетшин Ф.Ф. Динамическая модель штанговой насосной установки при одновременнораздельной эксплуатации // Проблемы сбора, подготовки и транспорта нефти и нефтепродуктов. - 2018. - № 3. - С. 33-41.

22. Методика расчета подвески плунжера в цилиндре насоса / Р.Н. Бахтизин, К.Р. Уразаков, Р.И. Бакиров, И.Р. Баширов, А.В. Киселев и др. // Нефтяное хозяйство. - 2018. - № 2. C. $84-88$

Поступила 24.01.2020 г. 
Известия Томского политехнического университета. Инжиниринг георесурсов. 2020. Т. 331. № 2. 188-198 Бахтизин Р.Н. и др. Двухуровневый метод диагностики технического состояния штанговых насосных установок

Информация об авторах

Бахтизин Р.Н., доктор физико-математических наук, профессор, ректор Уфимского государственного нефтяного технического университета.

Уразаков К.P., доктор технических наук, профессор, профессор кафедры машин и оборудования нефтегазовых промыслов Уфимского государственного нефтяного технического университета.

Исмагилов С.Ф., аспирант кафедры разработки и эксплуатации нефтегазовых месторождений Уфимского государственного нефтяного технического университета.

Давлетшин Ф.Ф., аспирант кафедры геофизики Физико-технического института Башкирского государственного университета. 
UDC 622.276 .53

\title{
TWO-LEVEL METHOD FOR DIAGNOSING TECHNICAL CONDITION OF SUCKER-ROD PUMPING UNITS
}

\author{
Ramil N. Bakhtizin 1 , \\ Ramil_Bahtizin@mail.ru \\ Kamil R. Urazakov ${ }^{1}$, \\ Urazakk@mail.ru \\ Salavat F. Ismagilov', \\ salavatf@mail.ru \\ Filyus F. Davletshin², \\ felix8047@mail.ru \\ 1 Ufa State Petroleum Technological University, \\ 1, Kosmonavtov street, Ufa, 450062, Russia. \\ 2 Bashkir State University, \\ 32, Validi street, Ufa, 450076, Russia.
}

Relevance. A significant number of the largest oil fields in Russia are in the final stages of development, characterized by a decrease in production volumes, increase in the share of complicated wells. Sucker-rod pump units are one of the most common methods of operating wells short-term production fund. The operation of rod pump installations in complicated operating conditions in some cases is accompanied by decrease in the turnaround time of work, increase in the specific energy and economic costs during production. In these conditions, one of the most urgent task is ensuring the cost-effective development of wells due to the timely diagnosis of the technical condition and operating conditions of pumping equipment.

Objects: pumping unit operating in various operating conditions, including in the presence of complicating factors, and a wellhead dynamometer card of operation of the rig, reflecting technical condition and operating conditions of the downhole equipment.

The aim of the research is to develop a new approach to diagnosing the state of rod pumping units using a dynamometer card, based on solving inverse problems of the dynamics of a rod installation.

Methods: methods for numerical solution of partial differential equations for direct problems, including modeling the theoretical dynamometer cards of the rod installation; methods for solving inverse problems aimed at quantifying the parameters of the model characterizing the operation of the rod installation

Results. The authors have developed two-tier method for diagnosing the state of pumping units using a dynamometer card. The proposed diagnostic method includes: at the first level, the processing of practical dynamometer cards by the pattern recognition system and, at the second level, a quantitative determination of the consequences of faults, based on the physical laws of formation of configuration of dynamometer cards, the solution of the problem of the dynamics of a rod installation. By analyzing the configuration of dynamometer cards under various operating conditions of pumping equipment (normal operation, high gas content at the pump intake, leakage in the pump discharge valve, low plunger fit in the cylinder), the examples of solving quantitative diagnostics problems based on the developed algorithm are shown.

Key words:

Rod pump installations, dynamometer card, modeling of dynamometer card, diagnostics, intake pressure, complicating factors, quantitative assessment.

\section{REFERENCES}

1. Aliev T.A., Rzayev A.H., Guluyev G.A., Alizada T.A., Rzayeva N.E. Robust technology and system for management of sucker rod pumping units in oil wells. Mechanical Systems and Signal Processing, 2018, vol. 99, pp. 47-56.

2. Boyuan Zh., Xianwen G. Sucker rod pumping diagnosis using valve working position and parameter optimal continuous hidden Markov model. Journal of Process Control, 2017, vol. 59, pp. 1-12.

3. Gilaev G.G., Efimenko B.V., Goncharova L.S., Isupov V.S. Diag nostirovanie glubinno- nasosnykh skvazhin dinamometrirovaniem [Diagnostics of deep-well wells with dynamometer]. Izhevsk, Paracelsus RA LLC Publ., 2008. 212 p.

4. Sadov V.B. The approach to the definition of the installation of downhole rod pump installations by a dynamogram. Oil industry, 2016, no. 2, pp. 90-93. In Rus.

5. Galdir D., Reges Jr., Schnitman L., Reis R., Mota F. A new approach to diagnosis of sucker rod pump systems by analyzing segments of downhole dynamometer cards. SPE (Society of Petro- leum Engineers) - 173964, SPE Artificial Lift Conference. Salvador, Bahia, Brazil, 27-28 May 2015. pp. 1-13.

6. Kovshov V.D., Sidorov M.E., Svetlakova S.V. Dynamometer, modeling and diagnostics of the state of a deep rod pumping unit. Izvestiya vuzov. Oil and gas, 2011, no. 3, pp. 25-29. In Rus.

7. Li K., Han Y., Wang T. A novel prediction method for down-hole working conditions of the beam pumping unit based on 8-directions chain codes and online sequential extreme learning machine. Journal of Petroleum Science and Engineering, 2018, vol. 160, pp. 285-301.

8. Sibagatullin R.R., Maysky R.A. Generalized algorithm for diagnosing the operation of shtangovye installations. Problems of collection, preparation and transport of oil and petroleum products, 2017, no. 3, pp. 69-77. In Rus.

9. Kuzmin A.N., Vyalykh I.A. Prediction of the technical condition of sucker rod pumps based on neural network technology. Bulletin of the Perm National Research Polytechnic University. Chemical technology and biotechnology, 2016, no. 3, pp. 9-19. In Rus. 
10. Li K., Xianwen G., Zhongda T., Zhixue Q. Using the curve moment and the Pso-Svm method to diagnose downhole conditions of a sucker rod pumping unit. Petroleum Science, 2013, vol. 10, pp. 73-80.

11. Li K., Gao X.W., Zhou H.B., Han Y. Fault diagnosis for downhole conditions of sucker rod pumping systems based on the FBHSC method. Journal of Petroleum Science and Engineering, 2015, vol. 12, pp. 135-147.

12. Virnovsky A.S. Teoriya i praktika glubinno-nasosnoi dobychi neft [Theory and practice of downhole oil production]. Moscow, Nedra Publ., 1971. $183 \mathrm{p}$.

13. Gibbs S.G., Neely A.B. Computer diagnosis of down-hole conditions in sucker rod pumping wells. Journal of Petroleum Technology, 1966, vol. 1, pp. 93-98.

14. Chen Z., White L.W., Zhang H. Predicting sucker-rod pumping systems with Fourier series. SPE (Society of Petroleum Engineers) Production \& Operations, 2018, vol. 33, pp. 928-940.

15. Zhao R., Peng, Y., Li Q., Shi J. Deep well lifting new solution in Tarim oilfield. SPE (Society of Petroleum Engineers) - 192494, SPE Middle East Artificial Lift Conference and Exhibition. Bahrain, 28-29 November 2018. pp. 1-14.

16. Li-Ming L., Hua Zh. Application and effect of buoyancy on sucker rod string dynamics. Journal of Petroleum Science and Engineering, 2016, vol. 146, pp. 264-271.

\section{Information about the authors}

Ramil N. Bakhtizin, Dr. Sc., professor, Ufa State Petroleum Technological University.

Kamil R. Urazakov, Dr. Sc., professor, Ufa State Petroleum Technological University.

Salavat F. Ismagilov, post-graduate student, Ufa State Petroleum Technological University.

Filyus F. Davletshin, post-graduate student, Bashkir State University. pp. 84-88. In Rus.
17. Hansen B., Tolbert B., Vernon C., Hedengren J.D. Model predictive automatic control of sucker rod pump system with simulation case study. Computers and Chemical Engineering, 2019, vol. 121, pp. $265-284$.

18. Bakhtizin R.N., Urazakov K.R., Ismagilov S.F., Topol'nikov A.S., Davletshin F.F. Dynamic model of a Rod Pump Installation for inclined wells. Socar Proceedings, 2017, no. 4, pp. 74-82.

19. Urazakov K.R., Bakhtizin R.N., Ismagilov S.F., Topolnikov A.S. Calculation of theoretical dynamograms taking into account complications in the operation of a well pumping pump. Oil Industry, 2014, no. 1, pp. 90-93. In Rus.

20. Brill J.P., Mukherjee H. Multiphase flow in wells. Richardson, Texas, Society of petroleum engineers, 1999. $384 \mathrm{p}$.

21. Urazakov K.R., Belov A.E., Davletshin F.F. Dynamic model of a rod pump installation for dual completion. Problems of collecting, preparing and transporting oil and oil products, 2018, no. 3, pp. 33-41. In Rus.

22. Bakhtizin R.N., Urazakov K.R., Bakirov R.I., Bashirov I.R., Kiselev A.V. et al. Method for calculating the plunger hanger in the cylinder of the sucker-rod pump. Oil Industry, 2018, no. 2,

Received: 24 January 2020. 